\title{
Rapid HIV testing program implementation: lessons from the emergency department
}

\author{
Christian Arbelaez • Brian Block • Elena Losina • Elizabeth A. Wright • \\ William M. Reichmann • Regina Mikulinsky • Jessica D. Solomon • \\ Matthew M. Dooley $•$ Rochelle P. Walensky
}

Received: 10 October 2008 / Accepted: 12 July 2009 / Published online: 1 September 2009

(C) Springer-Verlag London Ltd 2009

\begin{abstract}
Background The US Centers for Disease Control and Prevention (CDC) guidelines and the World Health Organization (WHO) both recommend HIV testing in health-care settings. However, neither organization provides prescriptive details regarding how these recommendations should be adapted into clinical practice in an emergency department. Methods We have implemented an HIV-testing program in the ED of a major academic medical center within the scope of the Universal Screening for HIV Infection in the Emergency Room (USHER) Trial - a randomized clinical trial evaluating the feasibility and cost-effectiveness of HIV screening in this setting.
\end{abstract}

The views expressed in this paper are those of the author(s) and not those of the editors, editorial board or publisher.

\footnotetext{
C. Arbelaez $(\bowtie) \cdot$ B. Block $\cdot$ J. D. Solomon $\cdot$ M. M. Dooley

Department of Emergency Medicine,

Brigham and Women's Hospital,

75 Francis St,

Boston, MA 02115, USA

e-mail: carbelaez@partners.org

E. Losina $\cdot$ E. A. Wright $\cdot$ W. M. Reichmann · R. Mikulinsky

Department of Orthopedic Surgery,

Brigham and Women's Hospital,

75 Francis St,

Boston, MA 02115, USA

R. P. Walensky

Division of Infectious Diseases, Department of Medicine,

Brigham and Women's Hospital,

75 Francis St,

Boston, MA 02115, USA

\section{R. P. Walensky}

Division of Infectious Disease, Department of Medicine,

Massachusetts General Hospital,

75 Francis St,

Boston, MA 02115, USA
}

Results and conclusion Drawing on our collective experiences in establishing programs domestically and internationally, we offer a practical framework of lessons learned so that others poised to embark on such HIV testing programs may benefit from our experiences.

Keywords HIV · Testing · Screening ·

Emergency department

\section{Introduction}

In the US, approximately $25 \%$ of the estimated 1 million people living with HIV infection are unaware of their

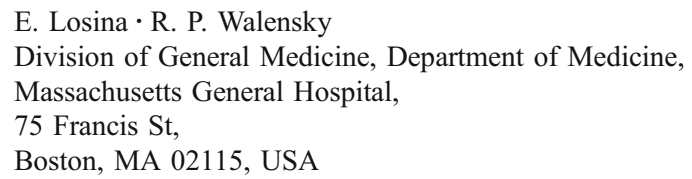

R. P. Walensky

Center for AIDS Research,

Harvard Medical School,

75 Francis St,

Boston, MA 02115, USA 
diagnosis. In response to these statistics, the Centers for Disease Control and Prevention (CDC) and the US Preventive Services Task Force (USPSTF) have offered recommendations on HIV testing. The CDC suggests that voluntary HIV testing be offered to adults ages 13-64 in all health-care settings, including the emergency department (ED) [2]. In contrast, the USPSTF strongly recommends that clinicians screen all adolescents and adults at increased risk for HIV infection, but makes no recommendation for or against routine screening [3]. Routine HIV testing in the Emergency Department is specifically mentioned in the CDC guidelines because of the tendency of the ED to serve a patient population who under-report their HIV risk and may not undergo risk-based HIV testing in other healthcare settings. In one study, $41 \%$ of these late-testers were diagnosed with AIDS within 1 year, and $79 \%$ of them had other health-care visits with a diagnosis that would not have prompted an HIV test under a risk-based approach. A targeted testing program may result in missed opportunities to screen many eligible individuals [4-6].

Without identification, HIV-infected patients lack access to life-saving therapies and continue to transmit disease. The American College of Emergency Physicians (ACEP) responded to the CDC recommendations with an HIV policy statement: "HIV screening must be: practical, feasible, not interfere with the primary acute care mission, based on the local prevalence, and integrated with the local health-care system" [7]. Since then, numerous approaches to HIV testing in the ED and ambulatory settings have already been implemented with varying degrees of reported success, measured by rates of test offering, test acceptance, receipt of test results, false-positive test results, cases identified, and confirmed linkage to appropriate care [8-23].

We designed and implemented an HIV testing program within the Universal Screening for HIV Infection in the Emergency Room (USHER) Trial, a randomized clinical trial conducted at a tertiary care center intended to examine ED provider-based testing versus HIV counselor-based testing. As an NIH-funded Trial, USHER examined numerous "best practice" approaches. In the implementation of such a program, there are many things to consider, including staff engagement, test selection, protocol development, education and training of personnel, and linkage to care. Our objective is to describe the development and implementation strategies of a large-scale routine HIV testing program in the ED so that others poised to embark on such programs may benefit from what we learned.

\section{Getting started}

The success of any ED-based HIV testing program relies heavily on the investment of a designated "champion(s)" who will lead the efforts within the ED community and hospital. As efforts often mirror compensation, this leadership role might ideally be a funded effort as a fraction of a full-time employee (FTE). The program's success will very much rely on the passion that this person conveys toward the HIV testing mission. His/her capacity for leadership, action, and consensus within all aspects of the ED staff will likely portend the program's success.

A needs assessment within the community and the patient population is also a helpful early step. What is the HIV prevalence within the hospital catchment area? At that prevalence, is there an unmet need for HIV testing services? It is also helpful to understand the referral base for patients who might be identified within the ED testing program. For the newly identified HIV-infected patients, what is the wait time for a new patient appointment? Is there clinical HIV outpatient capacity to longitudinally care for them? Finally, the implementation of such a program is largely facilitated by dedicated funding support prior to its implementation. During the needs assessment and analysis of current services, the possible funding mechanisms for the project may become evident. Can this information be shared with hospital administrators to indicate a need for dedicated resources toward the program? Draft budget proposals should consider necessary resources for personnel (e.g., counselors, social workers), HIV test kits, training sessions (often run by consultants), and confirmatory laboratory expenses (if not covered under clinical care). The short- and long-term sustainability of the HIV testing program often relies on a dedicated funding source.

Early in the program development, it is helpful to engage a comprehensive team required to ensure the necessary support for success. The hospital administration leadership, representatives from public affairs, and legal counsel may facilitate funding, issues that might draw lay press attention, and consent processes. Legal counsel may offer further invaluable assistance to ensure compliance with state and hospital regulations on patient confidentiality, name-based reporting to the state, medical liability, patient record documentation, and contractual issues.

\section{Emergency department providers}

The implementation of HIV testing within the ED impacts all ED staff including departmental leadership, faculty, residents, nurses, staff, social workers, and interpreters. As such, each provider's engagement, training, and understanding of their motivation is crucial to embark on such an effort. When applicable, it might be helpful to approach the leaders of each staff group individually (e.g., chair, clinical director, residency director, nurse manager, and assistant nurse manager) because of the differing impact the program may have on their respective employees. In order to remain 
focused on the primary ED mission, HIV screening efforts should be integrated with careful priority to patient care and flow. The goal is therefore to assimilate the program within (rather than layered on top of) the current standard ED processes. If directly involved in the testing program, each employee will want to know his/her expected role and its effect on his/her current patient care responsibilities. Consider requesting feedback from staff to modify the testing protocol appropriately.

Infectious disease providers

Critical to the mission of an HIV testing program is the capacity to link newly identified patients to care. As such, the HIV testing program should be handled in close collaboration with the infectious disease (ID) division that will often provide the patient's first follow-up visit. Early discussions with ID collaborators often include issues related to capacity (are there enough providers to serve newly identified patients?) and linkage to care (how soon can a patient identified in the program get a "new patient" appointment?). The ID division often has helpful resources that might be useful in the development of such protocols, creating synergy within the collaboration.

\section{Laboratory services personnel}

The involvement of the clinical laboratory can help facilitate compliance with HIV testing regulations. Engaging laboratory services early will assist with decisions regarding choice of tests (attributes and drawbacks of each). It may be valuable to seek their expertise on important items such as point of care testing, quality control measures, and HIV confirmatory procedures. A rapid, reliable, and laboratory compliant test should be the goal.

Patient satisfaction and flow in the ED often rely on turn-around times for test results (both initial and confirmatory); this may also be an important component of the discussion with laboratory services.

\section{The choice of tests}

In selecting the most appropriate HIV test, the following factors should be taken into consideration: health-care setting, HIV prevalence, current hospital standards, costs and available resources, laboratory preference, space available to conduct point-of-care testing, test performance, and turn-around time for results. This decision should be made in the context of the initial program design, patient flow, and previous experience and/or contracts with these manufacturers, which may help facilitate staff training. Since the use of other point of care tests, such as fingerstick glucometers, have been helpful in expediting care, a rapid HIV test may be the best test to use in a busy health-care facility [24].

As decisions are being made regarding which test to use, it is helpful to know the landscape of new Food and Drug Administration (FDA)-approved rapid HIV screening tests [22]. The FDA defines Clinical Laboratory Improvement Amendment (CLIA)-waived tests as simple laboratory examinations that use unprocessed specimens (whole blood or oral fluid) and have an insignificant risk of yielding an erroneous result $[25,26]$. The FDA requires that sites using CLIA-waived rapid HIV tests have a quality assurance (QA) program in place. CLIA-waived tests are well suited for use in the US because they can be performed by individuals without formal training in clinical and nonclinical sites. Furthermore, these tests offer patients negative or preliminary positive test results during the course of their visit [26]. The decision to use a point-of-care test will require a designated laboratory space in the ED, personnel training, strict QA measures, and meticulous documentation. The advantages of point-of-care HIV testing are decreased transit time, faster overall turnaround time, and high frequency with which patients receive their test results. These advantages should be weighed against the responsibility of strict laboratory standard compliance [26].

There are six rapid tests available on the market. These include: Oraquick ${ }^{\circledR}$ ADVANCE ${ }^{\mathrm{TM}}$ Rapid HIV 1/2 Antibody Test (Orasure Technologies, Inc., Bethlehem, PA); Uni-GoldTM Recombigen (B) HIV (Trinity Biotech, Bray, Ireland); Reveal G-3 Rapid HIV-1 (MedMira Laboratories, Inc., Halifax, Nova Scotia, Canada); MultiSpot ${ }^{\mathrm{TM}} \mathrm{HIV}-1 /$ HIV-2 test (BioRad Laboratories, Redmond, WA); Clearview ${ }^{\circledR}$ HIV 1/2 Stat-Pak ${ }^{\circledR}$; Clearview ${ }^{\circledR}$ HIV $1 / 2$ (Chembio Diagnostic Systems, Inc., Medford, NY). The reported sensitivity and specificity for the rapid FDA CLIA-waived HIV tests approach 100\%. Oraquick has a sensitivity $(95 \% \mathrm{CI})$ of $98.4-99.9 \%$ and a specificity $(95 \%$ CI) of $99.6-100 \%$. Uni-Gold has a sensitivity (95\% CI) of 99.5-100\% and a specificity (95\% CI) of $99.0-100 \%$. Reveal G-3 and MutiSpot are not CLIA-waived. Clearview has a sensitivity $(95 \% \mathrm{CI})$ of $98.9-100 \%$ and a specificity (95\% CI) of 99.6-100\% [25, 26]. Higher than expected rates of false-positivity have been reported, as reflected in lower positive predictive values in areas of low HIV prevalence [23, 27]. A preliminary positive test result requires confirmation by a more specific test, typically the Western blot. In one Boston-based ED experience, patients with a reactive oral Oraquick HIV test had an 8- to 32-fold increased odds of HIV infection compared with the pre-test odds, despite a lower than anticipated test specificity [23]. The goal for HIV testing programs is to optimize the test for the specific setting without compromising test reliability for the patients [1, 26-29]. 
Specimen collection

Test choice will determine the kind of specimen required to conduct the test. The specimen collection options for rapid HIV tests are oral fluid, serum fingerstick, and venipuncture. Test performance may vary by type of specimen collected, and understanding the performance characteristics of the test as they relate to specimen type is essential [23]. Furthermore, different collection options may affect patients' willingness to be tested, where the test can be developed, and the time that the patients will have to wait for results [26, 30, 31].

Test kit reading window period

The majority of currently available rapid HIV tests require that the test be conducted within 5 min of specimen collection. This requirement necessitates that the test be developed at the point of care, as there may be insufficient time to deliver the specimen to a central laboratory. Once the development process begins, however, tests also vary in their reading windows. For example, the OraQuick test takes a minimum of 20 min to develop, but may be read within a $20-40 \mathrm{~min}$ reading window. A longer reading window might enable a tester to step away, attend to another issue, and return to read the result. Another test, Reveal G-3 Rapid HIV-1, takes 3-5 min to conduct, but should be read immediately. This key test feature is helpful when considering the program design of whom the test operators will be and how their other responsibilities might distract them from reading the test result during the valid window. A test result that is not read within the specified time window should be considered invalid and repeated.

Procedures for ordering and receiving HIV test kits and controls

In a routine testing program, there is rapid test kit turnover. Systems should be considered to ensure adequate kit/ control supplies and that they are utilized within their designated shelf-life (kit/control shelf-life differs by manufacturer but is generally $6-24$ months). When choosing a test kit, it is helpful to engage the manufacturer to understand how the kits and controls are packaged (box size), how they must be stored (some require refrigeration and/or other temperature specifications), what the shelf-life is for each, and what control practices are suggested. Most manufacturers will recommend that with the arrival of new kits, expiration dates should be examined and quality assurance tests performed. Thus, having personnel responsible to receive these kits and perform these tasks is helpful.
College of American Pathology (CAP) certificate

In the US, a certification and quality assurance process exists (which is adhered to by standard clinical laboratories) for programs that choose point-of-care testing. To ensure that laboratory tests such as rapid HIV tests are being performed and interpreted correctly, the College of American Pathology (CAP) requires that test operators register for a certification process and participate in an external assessment of testing proficiency every 6 months [32]. This process serves as an external control for the rapid HIV testing conducted in the ED and is most often US hospital policy. The certificate is needed prior to program initiation and should be kept in the laboratory area.

\section{Quality assurance measures}

Quality management systems should be established in all settings performing HIV testing in order to validate standard operating procedures and confirm that testing algorithms are aligned with national guidelines [1]. These internal quality assurance measures help to ensure that the laboratory tests are reliable and are performing according to the manufacturers' expectations. HIV test controls should be specific to both manufacturer and test kit.

\section{HIV test costs}

Testing costs should be considered during budget preparation. The price per kit of rapid HIV tests ranges from \$14$\$ 25$ and may depend, in part, on negotiated hospital laboratory agreements. The costs associated with the controls and shipments of supplies are generally comparatively small. Patient volume and confirmatory algorithms should also be considered [1].

\section{Education and training}

\section{Training ED staff}

Obtaining "buy-in" from the ED staff is most successful when they are knowledgeable about the benefits of a routine HIV testing program. Research suggests that ED health-care providers generally feel uninformed about HIV testing and welcome more information and resources on the topic $[8,33]$. ED staff participants may enjoy meeting team members and learning about the program, including the testing protocols and patient resources. As an incentive, Continuing Education Units for nurses who attend the program may be awarded. Training sessions may include basic facts about HIV transmission, local incidence/prevalence estimates, and state-based ethical and legal issues surrounding HIV testing. 
HIV-related resources may be distributed including local HIV testing sites and HIV-related websites. A program website, a bulletin board, and a "frequently asked question" handout may be used to supplement communication with staff.

\section{Counseling and testing training of staff}

Many departments of public health offer a course to certify HIV counselors with training in the process of offering, consenting, testing, and counseling patients prior to HIV testing. The training for HIV Counseling, Testing, and Referral (CTR) is a useful resource where training participants receive a state-issued HIV counselor certificate. Training helps those designated to conduct the test learn the proper method to obtain a specimen, develop the rapid HIV test, and ensure their competency in reading the results.

\section{Space constraints within the ED}

Because the ED may consist of urgent, non-urgent, fast track, and observation units, patient care rooms may not be always private.

Offering the test and ensuring privacy

HIV testing is a sensitive issue that should ideally be conducted in a private space. To respect the patients' right to privacy, every effort should be made to present the testing program information and informed consent in a private area. Patients may be approached in the waiting room, triage, or their patient care rooms. In situations where ensuring privacy is difficult, efforts should be made to use quiet voices or point to, rather than read, sensitive text, especially in cases of curtain barriers in place of walls. If the patient population is predominantly non-English speaking, having a translator available may diminish testing barriers.

Data collection and documentation in the testing program

Patients who consent for testing may inquire whether participation will affect their care, their health-care providers will be notified about their participation, and their test results will be added to their medical records. These questions should be addressed in the context of program development. Consider collecting basic patient demographic information such as age, gender, race/ethnicity, primary language, and if applicable, the reason for refusing to participate.

Informed consent

HIV testing should be accompanied by basic pre-test information to enable the patient to make an informed and voluntary testing decision [1]. In the US, states vary on their requirements for the HIV informed consent process [34]. Knowledge of the current state law is important so that these can be communicated back to the patient. Some states (e.g., Massachusetts and New York) require written informed consent while others require verbal consent with documentation that this was obtained (e.g., California). In response to the CDC guidelines, states are actively engaged in changing state laws, and thus this process is a "moving target" [34]. Regardless, all states now require that newly HIV-diagnosed persons be reported by name; notification of this policy to patients is generally part of the consent process [34].

Test result delivery

As with the test offer and consent process, patients should receive both non-reactive (negative) and reactive (preliminary positive) HIV test results privately. Every effort must be made to ensure patient confidentiality regarding HIV test results by limiting the number of health-care providers who are made aware of the patients' results.

\section{What to do when a rapid HIV test is reactive (positive)}

Reactive (positive) rapid HIV test result protocol

Reactive tests may often produce anxiety among the testers, staff, and patients. A prescriptive plan regarding what to do with a reactive result may allay such anxieties. A "Reactive (Positive) Result Packet" (including mock scripts) may help personnel with the delivery of results and provide patients with available resources for follow-up and linkage to care. Such a packet may lead to standardization of this process and minimizing confusion in the reactive result delivery process (Table 1).

Table 1 Contents of a reactive (preliminary positive) rapid HIV test result protocol packet

"How to deliver a reactive result" script

The rapid HIV test manufacturer package insert

General information sheet on HIV infection

Confirmatory test protocol

- Pre-printed laboratory requisition slips

Linkage-to-care protocol

- Person in charge of scheduling appointments

- Parking arrangements

- Maps to the location of the appointment

- Interpreter contact information 
Confirmatory testing protocol

If a rapid HIV test is used, reactive test results require confirmation. An HIV diagnosis should not be delivered based on rapid test results alone. The CDC recommends that reactive rapid tests be confirmed using an HIV Western blot $[2,29]$. To maximize the information the patient receives at his/her first follow-up appointment, consider adding both a CD4 cell count and HIV RNA (viral load) to the confirmation panel [23]. Recent research suggests that confirmatory blood work is most often completed when done in the ED [11].

\section{Linkage-to-care protocol}

The final critical piece of any comprehensive HIV testing program is linkage to long-term HIV care. An appointment may involve delivery of confirmatory blood test results and linkage to care for newly identified patients. To maximize follow-up rates, it is helpful to have a prescriptive plan in place that can be easily followed by staff and is convenient for the patients. The plan may include scheduling appointments, transportation and parking arrangements, provision of maps to the clinic, and interpreter availability when necessary. Patients are often most comfortable if offered an appointment soon after the availability of confirmatory results. When such results appear before the designated appointment, it may be helpful to schedule an interim phone call. For patients who fail to keep their appointments, a designated individual should make repeated attempts to discreetly reach the patient in person, by phone, and/or by mail.

Protocol for those who leave without test results

Even in the era of rapid HIV tests, occasionally patients will leave the ED without receiving their HIV test results. It is critical to have a plan addressing how these cases will be handled for both negative and reactive results. Similar to the linkage-to-care protocol, patients may be contacted by phone, letters, and/or requests to return to the hospital (especially for reactive cases or when a patient will be back for other reasons). All attempts to reach the patient and deliver test results should be carried out with the same principles of privacy and confidentiality as if the patient were present in the ED.

\section{Other issues to consider}

\section{Manual of Operating Procedures}

To keep protocols standard, and so that all shifts will have access, it may be helpful to develop a Manual of Operating Procedures (MOOP). This is a "live" document that details protocols and algorithms, contains copies of all forms and resources, and provides education and training materials. It serves as a reference for all personnel and should be reviewed by every new hire into the program.

Pilot testing

Prior to full program implementation, it may be helpful to pilot the testing program. During this pilot period, simulated scenarios will assist personnel with developing responses to challenging situations. Pilots may occur during busy and slow ED times and at all hours of the day. The feedback received during these pilot periods may prove invaluable during implementation.

Sustainability

To help the HIV testing program achieve long-term sustainability, it is important to incorporate as many aspects as possible of the program and its personnel in the daily operations of the ED. For example, components such as personnel hiring, education and training sessions, protocol changes, and purchasing should become part of the daily routine in the ED. Furthermore, frequent, perhaps quarterly, evaluation is helpful to examine data including offer rates, acceptance rates, test rates, newly identified cases, and, ideally, the number of patients linked to care. Such crude statistics are vital in demonstrating the benefits of the program to future funders.

\section{International implications}

In contrast to the US, the HIV epidemic internationally is more extensive and severe. Daily, approximately 6,800 people are infected with HIV, and more than 5,700 die because of inadequate access to comprehensive HIV care [35]. The impact of an HIV epidemic differs from country to country. While the majority of developed countries have low level HIV epidemics (defined by WHO and UNAIDS as an HIV prevalence not exceeding $5 \%$ in any subpopulation), a substantial number of developing countries have a generalized HIV epidemic (classified as an HIV prevalence of greater than $1 \%$ among pregnant women) [1]. According to the $\mathrm{WHO}$, the needs assessment and ultimately the selection of priority interventions and target populations should be based on an understanding of the country's epidemiology, health-care infrastructure, societal norms, political will, and available resources [1]. HIV testing, one of the WHO Priority Interventions, should target patients in health facilities, communities, and specialized outreach modalities such as mobile units [1]. Numerous international HIV testing programs have been implemented and report a 
high yield of HIV case identification [28, 36-38]. However, not all programs have been successful. A recent report of a Lesotho program highlights the critical importance of training, expansion of services goals, adequate funding, and administrative capacity [39].

Implementation of routine HIV testing programs should be tailored to the specific international setting conditions. In developed countries with low-level HIV epidemics, many components of the program described in this report could be directly applicable to specific developed country settings. The initiation of an HIV screening program will likely require efforts similar to those of the US domestic programs and may include: identifying a program "champion," performing a needs assessment, understanding the local culture and population demographics, assessing the capacity of the local health care to provide HIV treatment, obtaining buy-in from the local leadership, and procuring sustainable funding. In resource-limited settings with generalized HIV epidemics, additional unique issues such as program costs and test acceptability related to HIV stigma may inhibit such efforts.

Internationally, the choice of test manufacturer may be influenced by a multitude of factors including: local regulation by in-country ministries, heat stability, storage capacity, staff training, and quality assurance measures. While the WHO lists rapid HIV antibody tests as essential laboratory tests at health centers and district hospitals, confirmation with the "gold standard" serum EIA and Western blot is often impossible. Consequently, serial (or parallel) rapid tests are often conducted for confirmation $[1,28,40]$. In areas of low prevalence, test users should be aware of the possibility of false-positive results. In high-prevalence areas, administrators should be aware that false-negative results may also occur. Clinical suspicion in the face of a negative test should prompt secondary testing [40]. Most importantly, because of the numerous barriers to follow-up, HIV testing programs in developed and developing countries should emphasize linkage to care protocols that are coordinated among patients, urgent care physicians, and HIV providers.

\section{Conclusions}

Based on our domestic and international experiences, an HIV testing program in the ED is feasible with appropriate infrastructure and longitudinal support. Although proof is still needed that HIV screening will alter HIV prevalence and incidence estimates over time, we describe a comprehensive and practical set of implementation strategies for a rapid HIV testing program in the ED. We examine issues related to the engagement of personnel, test choice, space, training, and follow-up of reactive results, capitalizing on our collective experiences in program establishment internationally [28] and domestically in urgent care centers [41], inpatient settings [42], prisons [43], and now, emergency departments [23]. Long-term sustainability requires further research to examine ways of addressing funding sources for these programs, eliminate medico-legal barriers, and improve the access to care for those found to be HIV-infected.

Conflicts of interest None.

Funding sources Experiences leading to this review and efforts to write it were funded by the National Institute of Mental Health (R01 MH0735445, R01 MH65869) and the Doris Duke Charitable Foundation Clinical Scientist Development Award. ClinicalTrials. Gov: \#NCT00502944

\section{References}

1. World Health Organization (2009) Priority interventions HIV/ AIDS prevention, treatment and care in the health sector, February 2009. Available at: http://www.who.int/hiv/pub/priority_interven tions_web.pdf. Accessed February 27, 2009

2. CDC (2006) Revised recommendations for HIV testing of adults, adolescents, and pregnant women in health-care settings. MMWR 55:RR-14

3. USPSTF (2009) Screening for HIV recommendation statement, April 2007. Available at: http://www.ahrq.gov/clinic/uspstf/ uspshivi.htm. Accessed February 27, 2009

4. Rothman RE, Ketlogetswe KS, Dolan T, Wyer PC, Kelen GD (2003) Preventive care in the emergency department: Should emergency departments conduct routine HIV screening? A systematic review. Acad Emerg Med 10(3):278-285

5. CDC (2006) Missed opportunities for earlier diagnosis of HIV infection - South Carolina, 1997-2005. MMWR 55(47):1269-1272

6. Millen JC, Arbelaez C, Walensky RP (2008) Implications and impact of the new US Centers for Disease Control and Prevention HIV testing guidelines. Curr Infect Dis Rep 10(2):157-163

7. American College of Emergency Physicians (2007) HIV testing and screening in the emergency department. Ann Emerg Med 50 (2):209

8. Arbelaez C, Losina E, Wright E, Millen J, Dooley M, Reichmann W et al (2007) In: Universal HIV testing in the ED- can we do it? Understanding the barriers affecting providers' willingness to test. Poster Abstract at ACEP, Seattle; 2007

9. Borg KT (2007) To test or not to test? HIV, emergency departments, and the new Centers for Disease Control and Prevention guidelines. Ann Emerg Med 49(5):573-574

10. Brown J, Shesser R, Simon G (2007) Establishing an ED HIV screening program: Lessons from the front lines. Acad Emerg Med 14(7):658-661

11. Brown J, Shesser R, Simon G, Bahn M, Czarnogorski M, Kuo I et al (2007) Routine HIV screening in the emergency department using the new US Centers for Disease Control and Prevention guidelines: Results from a high-prevalence area. J Acquir Immune Defic Syndr 46(4):395-401

12. Calderon Y, Haughey M, Leider J, Bijur PE, Gennis P, Bauman LJ (2007) Increasing willingness to be tested for human immunodeficiency virus in the emergency department during off-hour tours: A randomized trial. Sex Transm Dis 34(12):1025-1029

13. Haukoos JS, Hopkins E, Eliopoulos VT, Byyny RL, Laperriere KA, Mendoza MX et al (2007) Development and implementation 
of a model to improve identification of patients infected with HIV using diagnostic rapid testing in the emergency department. Acad Emerg Med 14(12):1149-1157

14. Irvin CB, Flagel BT, Fox JM (2007) The emergency department is not the ideal place for routine HIV testing. Ann Emerg Med 49 (5): 722

15. Lyons MS, Lindsell CJ, Fichtenbaum CJ, Camargo CA Jr (2007) Interpreting and implementing the $2006 \mathrm{CDC}$ recommendations for HIV testing in health-care settings. Public Health Rep 122 (5):579-583

16. Lyss SB, Branson BM, Kroc KA, Couture EF, Newman DR, Weinstein RA (2007) Detecting unsuspected HIV infection with a rapid whole-blood HIV test in an urban emergency department. J Acquir Immune Defic Syndr 44(4):435-442

17. McKenna M (2007) HIV testing: Should the emergency department take part? Ann Emerg Med 49(2):190-192

18. Mehta SD, Hall J, Lyss SB, Skolnik PR, Pealer LN, Kharasch S (2007) Adult and pediatric emergency department sexually transmitted disease and HIV screening: Programmatic overview and outcomes. Acad Emerg Med 14(3):250-258

19. Rothman RE, Lyons MS, Haukoos JS (2007) Uncovering HIV infection in the emergency department: A broader perspective. Acad Emerg Med 14(7):653-657

20. Silva A, Glick NR, Lyss SB, Hutchinson AB, Gift TL, Pealer LN et al (2007) Implementing an HIV and sexually transmitted disease screening program in an emergency department. Ann Emerg Med 49(5):564-572

21. Branson B, Wesolowski L, Delaney K, Mavinkurve M, Dowling T, Mackellar D (2006) In: Investigation of reports of excessive false-positive oral fluid rapid HIV tests. Conference on Retroviruses and Opportunistic Infections; 2006; Denver, CO

22. Ehrenkranz PD, Ahn CJ, Metlay JP, Camargo CA Jr, Holmes WC, Rothman R (2008) Availability of rapid human immunodeficiency virus testing in academic emergency departments. Acad Emerg Med 15(2):144-150

23. Walensky RP, Arbelaez C, Reichmann WM, Walls RM, Katz JN, Block BL et al (2008) Revising expectations from rapid HIV tests in the emergency department. Ann Intern Med 149(3):153-160

24. Sands VM, Auerbach PS, Birnbaum J, Green M (1995) Evaluation of a portable clinical blood analyzer in the emergency department. Acad Emerg Med 2(3):176

25. Test waived by FDA from January 2000 to present. http://www. accessdata.fda.gov/scripts/cdrh/cfdocs/cfClia/testswaived.cfm. Last accessed on September 19, 2008. [Homepage on the Internet]

26. Greenwald JL, Burstein GR, Pincus J, Branson B (2006) A rapid review of rapid HIV antibody tests. Curr Infect Dis Rep 8(2):125131

27. CDC (2008) False-positive oral fluid rapid HIV tests-New York City, 2005-2008. Morb Mortal Wkly Rep 57(early release):1-5

28. Bassett IV, Giddy J, Nkera J, Wang B, Losina E, Lu Z, Freedberg KA (2007) Walensky RP. J Acquir Immune Defic Syndr 46 (2):181-186

29. Centers for Disease Control and Prevention (CDC) (2004) Notice to readers: protocols for confirmation of reactive rapid HIV tests. MMWR Morb Mortal Wkly Rep 53(10):221-222

30. Kshatriya R, Cachafeiro AA, Kerr RJ, Nelson JA, Fiscus SA (2008) Comparison of two rapid HIV assays, determine HIV-1/2 and OraQuick ADVANCE rapid HIV-1/2 for detecting recent HIV seroconversion. J Clin Microbiol, Aug 6

31. Syed Iqbal H, Balakrishnan P, Murugavel KG, Suniti S (2008) Performance characteristics of a new rapid immunochromatographic test for the detection of antibodies to human immunodeficiency virus (HIV) types 1 and 2. J Clin Lab Anal 22(3):178-185
32. College of American Pathologists. http://www.cap.org/apps/cap. portal. Last accessed on May 12, 2009. [Homepage on the Internet]

33. Arbelaez C, Losina E, Wright E, Millen J, Kimmel S, Dooley M et al (2008) Emergency provider attitudes and barriers to universal HIV testing in the ED. Oral Abstract at SAEM, Washington, DC

34. Bartlett JG, Branson BM, Fenton K, Hauschild BC, Miller V, Mayer KH (2008) Opt-out testing for human immunodeficiency virus in the United States: Progress and challenges. JAMA 300 (8):945-951

35. AIDS epidemic update. UNAIDS, WHO. 2007

36. Menzies N, Abang B, Wanyenze R, Nuwaha F, Mugisha B, Coutinho A, Bunnell R, Mermin J, Blandford JM (2008) The costs and effectiveness of four HIV counseling and testing strategies in Uganda. AIDS. 2008 Dec 24. [Epub ahead of print]

37. Solomon S, Venkatesh KK, Srikrishnan AK, Mayer KH (2008) Challenges of expansion of voluntary counselling and testing in India. Sex Health 5(4):371-372

38. Veloso VG, Portela MC, Vasconcellos MT, Matzenbacher LA, de Vasconcelos AL, Grinsztejn B, Bastos FI (2008) HIV testing among pregnant women in Brazil: rates and predictors. Rev Saude Publica 42(5):859-867

39. Daily HIV/AIDS report. Lesotho's HIV testing program has not met goals, group says. November 19, 2008. www.GlobalHealth Reporting.org. Last accessed on March 6, 2009

40. Bassett I, Chetty S, Giddy J, Reddy S, Losina E, Freedberg K, Walensky R (2008) False negative rapid HIV tests in Durban, South Africa. Poster Abstract at CROI, Boston, MA; 2008

41. Walensky RP, Losina E, Malatesta L, Barton GE, O'Connor CA, Skolnik PR et al (2005) Effective HIV case identification through routine HIV screening at urgent care centers in Massachusetts. Am J Public Health 95(1):71-73

42. Walensky RP, Losina E, Steger-Craven KA, Freedberg KA (2002) Identifying undiagnosed human immunodeficiency virus: The yield of routine, voluntary inpatient testing. Arch Intern Med 162 (8):887-892

43. Liddicoat RV, Zheng H, Internicola J, Werner BG, Kazianis A, Golan $Y$ et al (2006) Implementing a routine, voluntary HIV testing program in a Massachusetts county prison. J Urban Health 83(6):1127-1131

Christian Arbelaez is the Associate Director of the Office for Multicultural Careers at the Brigham and Women's Hospital (BWH) and the Assistant Residency Director of the Harvard Affiliated Emergency Medicine Residency Program at the BWH/MGH Hospitals.

Dr. Arbelaez has led a hospital-wide development, adoption, and implementation of guidelines for the care of the critically ill, mechanically ventilated patient, participated in the Hurricane Katrina Disaster Relief Efforts with the American Red Cross, and completed his first physician leadership book, "The Emergency Medicine Chief Resident Survival Guide." As a co-investigator of an RO1 NIHfunded study, he successfully implemented a large-scale clinical trial evaluating rapid HIV testing in the ED. Dr. Arbelaez has been an invited speaker nationally for his work with Workforce Diversity and HIV testing in the ED.

Before coming to the $\mathrm{BWH}$, he earned his medical degree from UTMB, completed his residency at the Brown Medical School Residency Program in Emergency Medicine, and earned his Master of Public Health from the Harvard School of Public Health as part of the Commonwealth Fund Minority Health Policy Fellowship. 